\title{
AUTOMATIC SOLAR TRACKING SYSTEM
}

\author{
Eduard-Valentin Canale ${ }^{1}$, Alexandru Stan ${ }^{1}$, Victor-Marin Zafiu ${ }^{1}$, Andrei Cristan Dinu ${ }^{1}$ \\ ${ }^{1}$ National Institute of Research and Development for Mechatronics and Measurement Technique - INCDMTM, \\ Bucharest \\ E-mails: eduard.canale@gmail.com, alexandrustan71.sa@gmail.com,victor.zaf@gmail.com, \\ andrei.dinu931@gmail.com
}

\begin{abstract}
The system presented in this article is based on the knowledge of a fixed solar panel does not have the maximum power output during the entire day because it is fixed at a given angle and a given heading. To increase the power output of a solar panel it needs to be in the direct path of the Sun all day. This can be done by moving on a single axis, which increases the efficiency of the system in the middle of the day, or by moving the system on two axes, the pan axis and the tilt axis, as the system described in this article. This kind of system ensures the max power efficiency at any time of the day.
\end{abstract}

Keywords: Solar Tracking, Solar Panel, Efficiency, Power Output.

\section{Introduction}

At the moment the push to save the planet with clean energy has skyrocketed, solar energy being the forefront of it. In 2017 all the solar systems have produced 2\% [1] of the entire global output and in 2018 that the percentile figure increased to almost $3.5 \%[2]$.

At the moment most of the solar energy is harvested by solar farms and home solar systems [3] that are fixed in position, only a few numbers of solar tracking system [4] are used to maximize the efficiency of the solar arrays.

In this article it will be presented an automatic solar tracking system based on a low-cost solution which integrates a microcontroller, an array of photo resistive sensors, a double $\mathrm{H}$-Bridge module and two geared motors for the movement of the solar panel.

\section{The Control System}

For the control system it was selected the AtMega 328 microcontroller as the "brain" of the system, it is a cheap and easy to work with microcontroller, it has the number of pins needed for all the desired connections, and it is very low power and long lasting.

The microcontroller is receiving the position of the Sun using four sensors that are positioned in a cross shaped jig (Fig. 1).

The sensors are connected to the microcontroller at the analogic inputs of it and are using the integrated ADC (Analog to Digital Convertor), with the resolution of 10 Bit giving data ranging from 0 to 1023 , using a grounded resistor as for each of the sensors to ensure no "phantom" readings by the sensors.

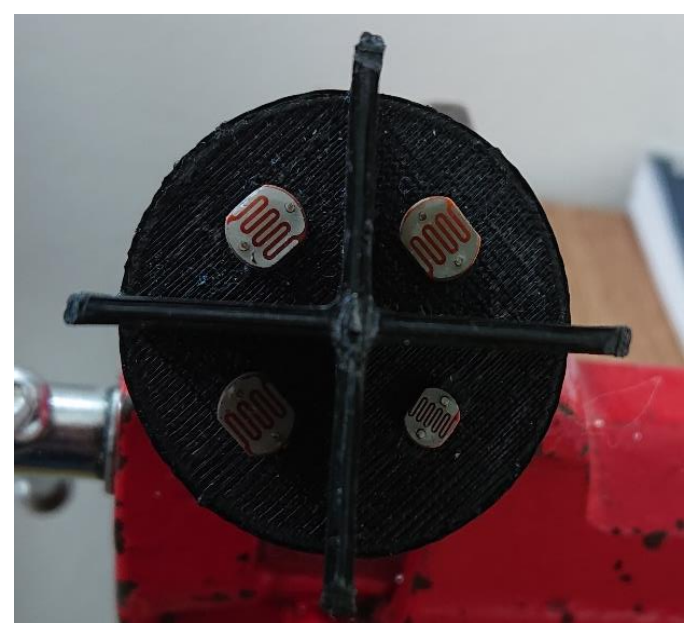

Figure 1: The 4 sensors and the cross shaped jig that holds them 
The information received from the sensors is transformed in ON or OFF signals for the motor that are transmitted to the dual $\mathrm{H}$-Bridge module that is based on the cheap L298 IC with a maximum output current of $2 \mathrm{~A}$ at a maximum voltage of $36 \mathrm{~V}$ (Fig. 2). The H-Bridge has a $5 \mathrm{~V}$ linear converter which ensures the power for the microcontroller and the four sensors.

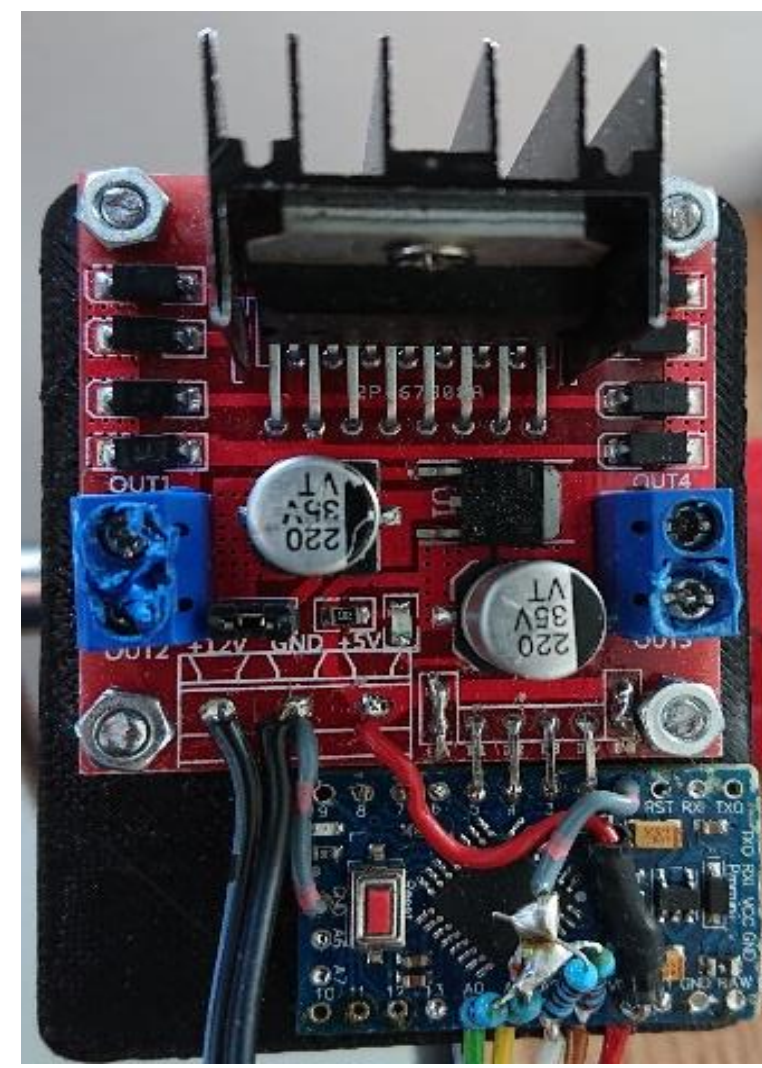

Figure 2: The H-Bridge with the red PCB and the microcontroller with the blue PCB

\section{The Movement System}

For the actuating of the solar panel two geared motors are used in a stacked one on top of the other at a 90-degree angle configuration. This configuration is the easiest way to give the system the two-axis needed track the sun. The pan-axis movement is ensured by a geared motor underneath that is connected to the small spur wheel on top. For the tilt-axis an enclosed planetary gear motor was used which is connected with the solar panel via a plate and with the pan motor via the second large spur wheel (Fig. 3).

Using this configuration, the automatic solar tracking system is capable of moving independently on both axes at any given time.

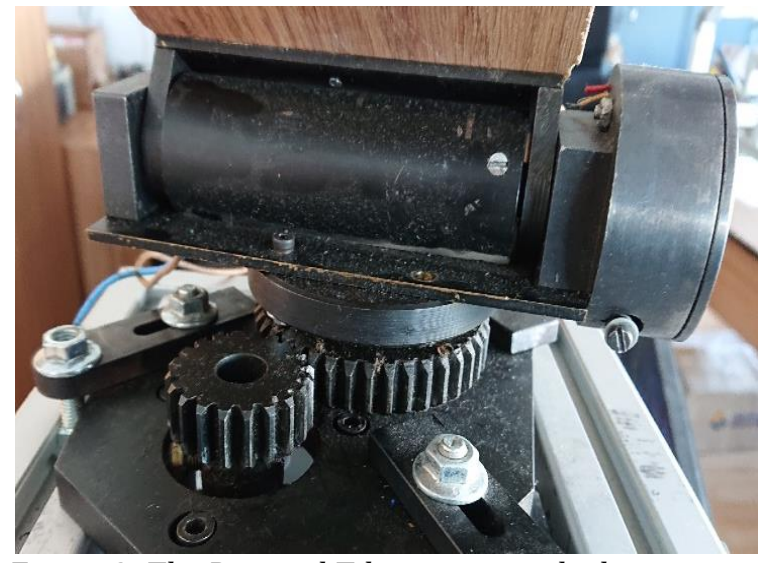

Figure 3: The Pan and Tilt system stacked one on top of the other

\section{The Harvesting and Transformation of Solar Energy into AC power}

At the heart of the solar energy harvesting is a $100 \mathrm{~W}$ solar panel which is rated at $22 \mathrm{~V}$ and $5 \mathrm{~A}$, with $110 \mathrm{~W}$ of peak power. The solar panel is connected to a solar MPPT, (Maximum Power Point Tracking), charge controller [5] which, as the name implies, it tracks how much power the system is getting from the solar panel at any time and by using the DC to DC convertor that transforms the higher voltage and lower current that comes out of the solar panel in a lower voltage and higher current for recharging the battery or powering on the other systems.

The charge controller is connected to a battery bank which in turn is connected to a DC to AC invertor for powering up a Laptop and the automatic solar tracking system (Fig. $4 a$ and $b$ ).

The components present in the system:

1 Enclosure for the MPPT controller, Invertor, Battery and for the microcontroller and $\mathrm{H}$ Bridge;

2 Invertor;

3 Pan motor;

4 Solar panel;

5 Photo-resistive sensor in the cross shaped jig

612 gel battery;

7 The command and control system, the $\mathrm{H}$ Bridge and microcontroller;

8 MPPT charge controller;

9 Laptop for plotting the amount of power made at a given time;

10 USB cable for the connection between the laptop and the MPPT charge controller;

11 Tilt motor;

12 230V AC invertor socket. 


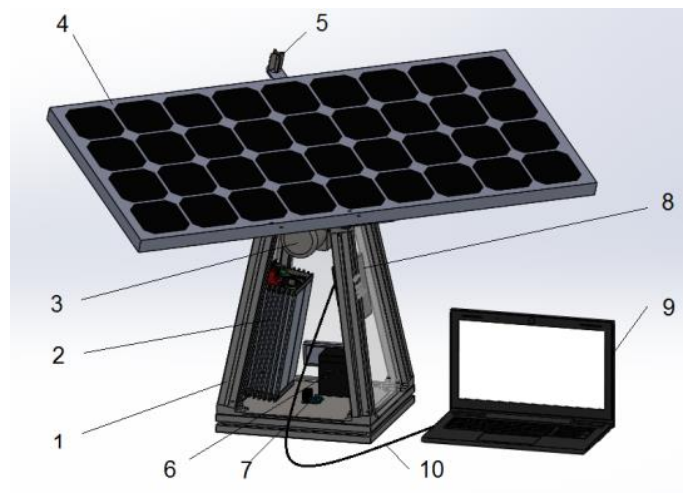

a)

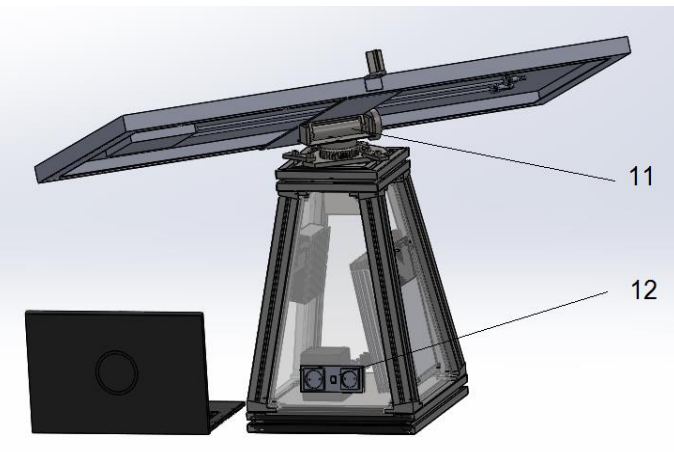

b)

Figure 4: The front and back 3D representations of the system

\section{The Control Algorithm for the Solar Tracking System}

The data received from each of the four photoresistive sensors is analogic data, a higher number meaning more light and a lower one a less light read by the sensor and it is different because the cross shaped walls of the jig are shadowing them more or less, depending on the relative position from the sun.

When the data received from the top two sensors is different from the data received from the ones that are the bottom the system is going to move in the direction of the of the sensors that receive more light.

The same principle is used for the pan, if the data from the right sensors is different from the data received from the left ones, the system is going to move in the direction of the more illuminated sensors. When the sensors are receiving the same amount of light, the system is stopped. By using this kind of algorithm, the system does not need a reset command for the night because the system is going to stop during the night and move in position at dawn when the Sun is going to cast a shadow on the sensors (Fig. 5).

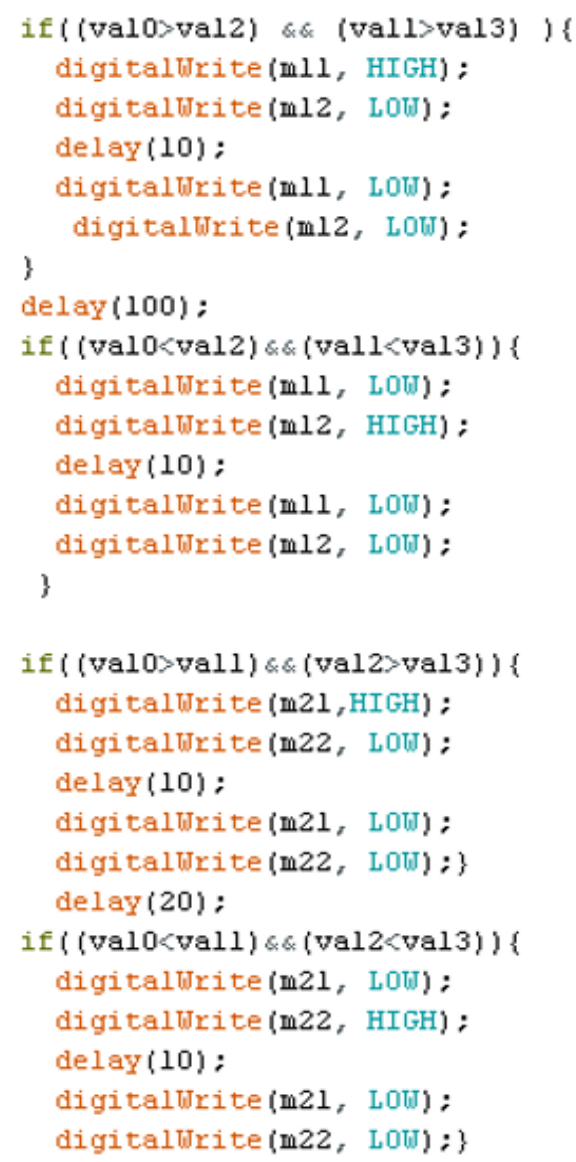

Figure 5: The control portion of the program used by the microcontroller 


\section{Energy Harvesting Efficiency and Power Consumption}

Taking into account the peak power produced by the solar panel of $110 \mathrm{~W}$ it can be calculated the maximum theoretical power for a 10 -hour day is about $1100 \mathrm{Wh}$ that can be used for charging the battery, powering the automatic solar tracking system and powering up the laptop.

The theoretical power is calculated taking into account the best-case scenario, no clouds, during the summer time and $100 \%$ power output from the solar panel and charge controller. Knowing that a solar panel has a lower efficiency once it gets hot, the percentile figure of the efficiency drops down to around $85 \%$, bringing down the power produced by the solar panel to around 90W, adding to this the efficiency of the charge controller and the integrated DC to DC converter of around 80\% [6] the maximum practical power produced by the entire system is around $750 \mathrm{Wh}$, meaning the system is producing an average of $75 \mathrm{~W}$ of power at any given moment of the day.

From the $75 \mathrm{~W}$ of produced power, the automatic solar tracking system is using around $12 \mathrm{~W}$ of power when the system moves and around 50W are used by the invertor that powers the laptop, meaning that the system is charging the system battery, powers up the laptop and the automatic solar tracking system at the same time.

The remaining $13 \mathrm{~W}$ of power that are used to charge the battery can theoretically charge a $12 \mathrm{Ah}$ battery from 0 , at the start of the day, to full in a 10 hour day, but taking into account that the system will not consume $62 \mathrm{~W}$ all the time, a $20 \mathrm{Ah}$ battery can be used as the main battery for the system ensuring enough power for the automatic solar tracking system in the morning and for the laptop during the night.

The power used by the automatic solar tracking system is made up by the fact that the system is producing the peak power at any given moment not only during the middle of the day, ensuring the constant $75 \mathrm{~W}$ of power from dawn to dusk.

Tests for the automatic solar tracking system were made during the summer with good results, the system was left outside for about 7 hours and during this time the solar tracking worked as intended and the system kept a $4 \mathrm{Ah}$ batter charged all the time even though it had to power the automatic solar tracking system (Fig. 6).

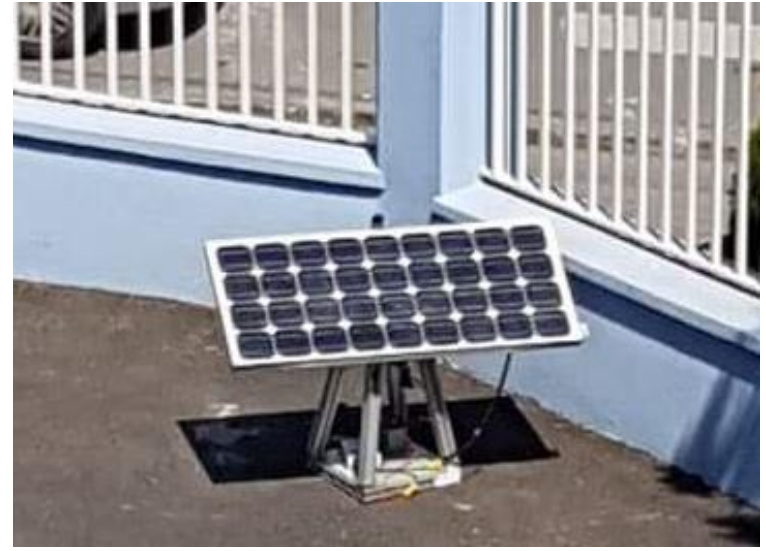

Figure 6: The system in a real-world test environment

\section{Conclusions}

The article is presenting an automatic solar tracking system used to increase the efficiency of a solar system by using a pan and tilt system based on two geared motors and a cheap control system.

The system is more complicated because of the added motors but the disadvantage brought by them is outweighed by the fact that the system efficiency is increased by around 20\% which ensures enough power for the automatic solar tracking system and the said laptop.

\section{Acknowledgements}

This work was supported by a grant of the Romanian Ministry of Research and Innovation, Research program NUCLEU, contract no. 17N/2019, project number PN 19.24.01.01.

\section{References}

[1] www.iea.org/topics/renewables/solar/ accessed at: $11 / 04 / 2019$

[2] en.wikipedia.org/wiki/Growth_of_photovoltaics accessed at: 11/04/2019

[3] Daniel B., Octavian D., Gheorghe GI., Constantin

[4] V., Alina S. Mechatronic system for solar energy

[5] acquisition. International Journal of

[6] Mechatronics and Applied Mechanics, 2017,

[7] 2017(1): pp. 75-80

[8] Rajan K. Solar Tracking System-A review. International Journal of Sustainable Engineering 2015

[9] www.solar-electric.com/learning-center/mpptsolar-charge-controllers.html/ accessed at: $11 / 04 / 2019$

[10] www.digikey.com/en/maker/blogs/introductio n-to-dc-dc-converters accessed at: 11/05/2019 